\title{
Hydrothermal Preparation of High Purity of Expandable Graphite
}

\author{
Yan $W^{1, a^{*}}$, Ji-Yang MA ${ }^{2, b}$, Bo ZHOU ${ }^{1, c}$, Yao-Qun HUANG ${ }^{1, d}$ \\ ${ }^{1}$ Engineering Research Academy of Graphite New Materials, Heilongjiang University of Science \\ and Technology, Harbin, 150022, PR China \\ ${ }^{2}$ College of Materials Science and Engineering, Heilongjiang University of Science and Technology, \\ Harbin, 150022, PR China \\ awuyanusth@163.com, b303721363@qq.com, capenbox@126.com, dhuangyaoqun@126.com \\ ${ }^{*}$ Corresponding author
}

Keywords: Hydrothermal, High Purity, Expandable Graphite.

\begin{abstract}
Hydrothermal preparation of expandable graphite, which is mixing natural flake graphite , oxidant and intercalating agent to hydrothermal reaction. Hydrothermal could improve the oxidicability and intercalating ability of oxidant and intercalate agent, ensuring the smooth progress of the oxide-intercalated reaction and reducing the phenomenon of 'micro-expansion' of expandable graphite. This essay made oxide-intercalated of natural flake graphite by hydrothermal to preparation of expandable graphite, examining the dosage of oxidant and intercalating agent, reaction time, reaction temperature and drying time in influence on the preparation of expandable graphite.
\end{abstract}

\section{Introduction}

Expandable graphite after high temperature treatment produced expanded graphite which with lubrication, high conductivity, good machining performance, adsorption and catalytic performance, sealing performance, flame retardant performance, and many other excellent performance, it has a wide application in industry[1-2]. Expandable graphite mainly has two kinds of preparation methods: chemical oxidation and electrochemical oxidation, and the chemical oxidation method is the most mature and in industrial application[3-6]. In the process of chemical oxidation, intercalating agent and oxidant's unreasonable choice, or too long reaction time can cause graphite peroxide phenomenon. The crystal structure of 'micro-expanded' expandable graphite was severely damaged with long time of expansible graphite oxidation, which then leads to the loose of graphite volume, product particle size will be smaller compared with raw graphite. This phenomenon becomes more severe with rise as oxidant of oxidizing and the extension of reaction time, which seriously influences the product of the expansion ratio and actual application.

Hydrothermal preparation of expandable graphite is mixed with natural flake graphite materials, oxidant, intercalating agent evenly, and put into high-pressure reaction kettle with teflon neck buch, with a suitable reaction temperature environment[7]. At the same time of high temperature reaction, it provides a suitable pressure for the system. In this way, it not only improved the oxidicability and intercalating ability of oxidant and intercalate agent, but also ensured the smooth progress of the oxide-intercalated reaction; and meanwhile in the case of various technological parameter's equal, the 'micro-expansion' degree of expandable graphite by hydrothermal has the phenomenon of obvious decrease.

This paper made oxide-intercalated of natural flake graphite by hydrothermal to preparation of expandable graphite, examining the dosage of oxidant and intercalating agent, reaction time, reaction temperature and drying time, and the influence on the preparation of expandable graphite. Through the single factor experiment method to study the influence factors on the impact of expandable graphite expansion ratio, optimizing the process parameters , then we determine the optimal experiment scheme. 


\section{Experiments}

\section{Materials}

Nature graphite were 100 mush which purchasd from Xinshun Graphite Ltd. Co. The graphite were purified by acid-alkali method, the carbon content were 99.93\%. Other reagents were of analytical grade and were used without further purification.

\section{Preparation of Expendable Graphite}

The typical experiment as 3 g graphite were mixed with $7 \mathrm{ml}$ concentrated sulfuric acid, adding different amount of hydrogen peroxide, after stirred $10 \mathrm{~min}$ were put into high-pressure reaction kettle with teflon neck buch and pass on to over with different temperature for hydrothermal. After a certain reaction time, the kettle was cooling to room temperature, collected the production by filter and washed by deionized water to the $\mathrm{pH}$ value was neutral. The samples were dried at $60 \mathrm{oC}$ in oven last $6 \mathrm{~h}$. The traditional chemical method to prepare expendable graphite was as literature[8].

\section{Characterization}

Expansion volume test was take as accurately according to $1.000 \mathrm{~g}$ of expandable graphite, put in quartz glass with scale and put into muffle furnace at $1000 \mathrm{oC}$ for 30 seconds, read the volume of the sample after expansion[9]. The morphology of sample was examined by scanning electromicroscope (SEM, JSE-6301F, JEOL).

\section{Results and Discussion}

\section{H2O2 Dosage Influence on Expansion Volume}

For examine the $\mathrm{H} 2 \mathrm{O} 2$ dosage influence on expansion volume, we take graphite $3 \mathrm{~g}$ graphite mixing $8 \mathrm{ml}$ of concentrated sulfuric acid, and adding different amount of hydrogen peroxide to preparation expendable graphite, reaction temperature is $80 \mathrm{oC}$ and reaction time $40 \mathrm{~min}$, the results are shown in Fig.1.

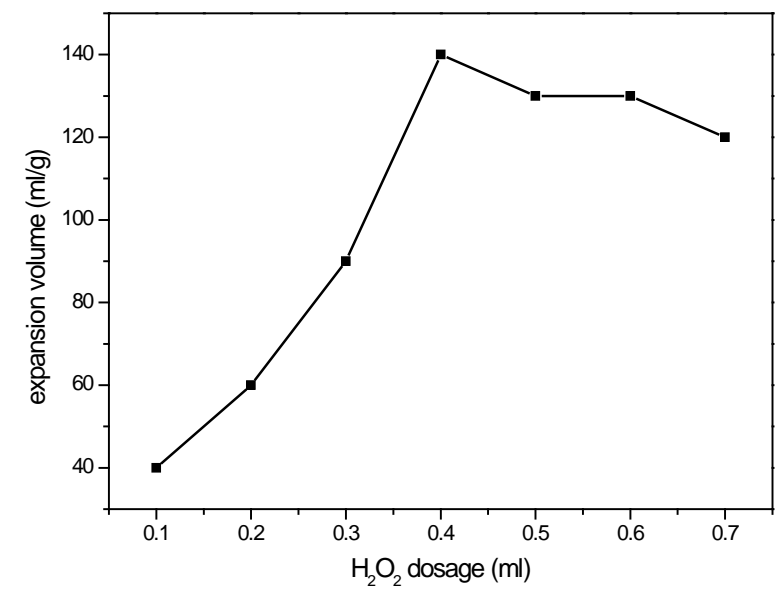

Fig. 1 The $\mathrm{H} 2 \mathrm{O} 2$ dosage influence on expansion volume

Along with the increase of hydrogen peroxide dosage, the preparation of the expansion volume of expansible graphite also increase, when the dosage of hydrogen peroxide was $0.4 \mathrm{~mL}$, as to achieve the maximum of expansion volume $140 \mathrm{ml} / \mathrm{g}$, continue to increase the dosage of hydrogen peroxide, expansion volume tends to be stable, no longer increases.Too much hydrogen peroxide will make expandable graphite peroxide occur, affecting its performance, therefore, after the comprehensive consideration on the whole experiment, selected $0.4 \mathrm{ml}$ for the experiments the optimum dosage of hydrogen peroxide.

\section{H2SO4 Dosage Influence on Expansion Volume}

Take $3 \mathrm{~g}$ of graphite and hydrogen peroxide $0.4 \mathrm{ml}$, adding different amount of sulfuric acid, reaction under $80 \mathrm{oC}$ for $40 \mathrm{~min}$ to rescarch the influence of the dosage of sulfuric acid on 
expansion volume, the results are shown in Fig. 2. From Fig.2 we can see that the effect of dosage of $\mathrm{H} 2 \mathrm{SO} 4$ on expansion volume is not large, with the increase of dosage of H2SO4, expansion volume is not much change. Continue to increase the dosage of H2SO4beyond $7 \mathrm{ml}$, expansion volume increases no longer, but has a tendency to reduce.Excessive dosage of H2SO4 without increasing volume expansion, at the same time also can make the product introduced a lot of sulfur, reduce the use of performance of the products.

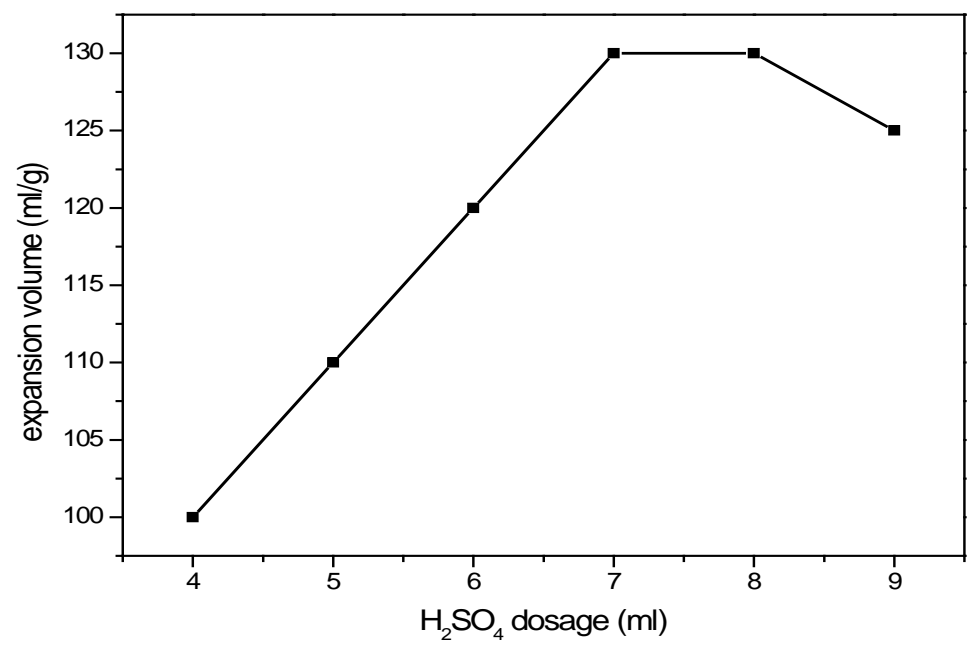

Fig.2 The H2SO4 dosage influence on expansion volume

\section{Reaction Temperature Influence on Expansion Volume}

$3 \mathrm{~g}$ graphite, $0.4 \mathrm{ml} \mathrm{H} 2 \mathrm{O} 2,7 \mathrm{ml} \mathrm{H} 2 \mathrm{SO} 4$ and reaction time is 40 min were taken to study the effect of reaction temperature on expansion volume and the results are shown in Fig. 3. The Fig.3 shows that, the expansion volume of $160 \mathrm{ml} / \mathrm{g}$ when the reaction temperature at $100 \mathrm{oC}$. Continue to rise reaction temperature, expansion volume fluctuate and change is not big. Therefore selected 100 oC for optimum reaction temperature.

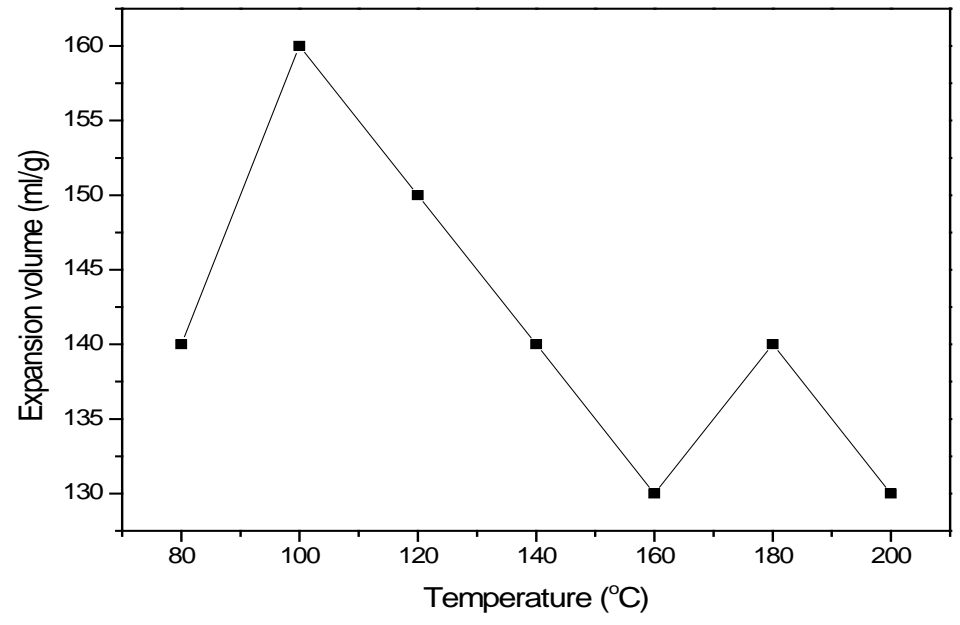

Fig.3 The temperature influence on expansion volume

\section{Reaction Time Influence on Expansion Volume}

3 g graphite, $7 \mathrm{ml} \mathrm{H} 2 \mathrm{SO} 4$ and $0.4 \mathrm{ml} \mathrm{H} 2 \mathrm{O} 2$ were reaction of different time under $100 \mathrm{oC}$ to study the effect of reaction time on expansion volume, the result is shown in table 1 . The expansion volume was achieve $160 \mathrm{ml} / \mathrm{g}$ when reaction time was $60 \mathrm{~min}$, and it was the optimal reaction time.

Tab. 3-3 The effect of reaction time on expansion volume

\begin{tabular}{lccccccc}
\hline Reaction time $(\mathrm{m} \mathrm{in})$ & 20 & 40 & 60 & 80 & 100 & 120 & 140 \\
\hline expansion volume $(\mathrm{ml} / \mathrm{g})$ & 120 & 140 & 160 & 160 & 150 & 120 & 130 \\
\hline
\end{tabular}




\section{The Morphology of Expandable Graphite}

The Fig.4 shows the morphology of nature graphite and expandable graphite. The nature graphite (a) was layers structure and the structure was integrated without oxide-intercalated reaction. (b) was the 'micro-expansion' expandable graphite, the edge of graphite was peroxide and has been slightly raised.
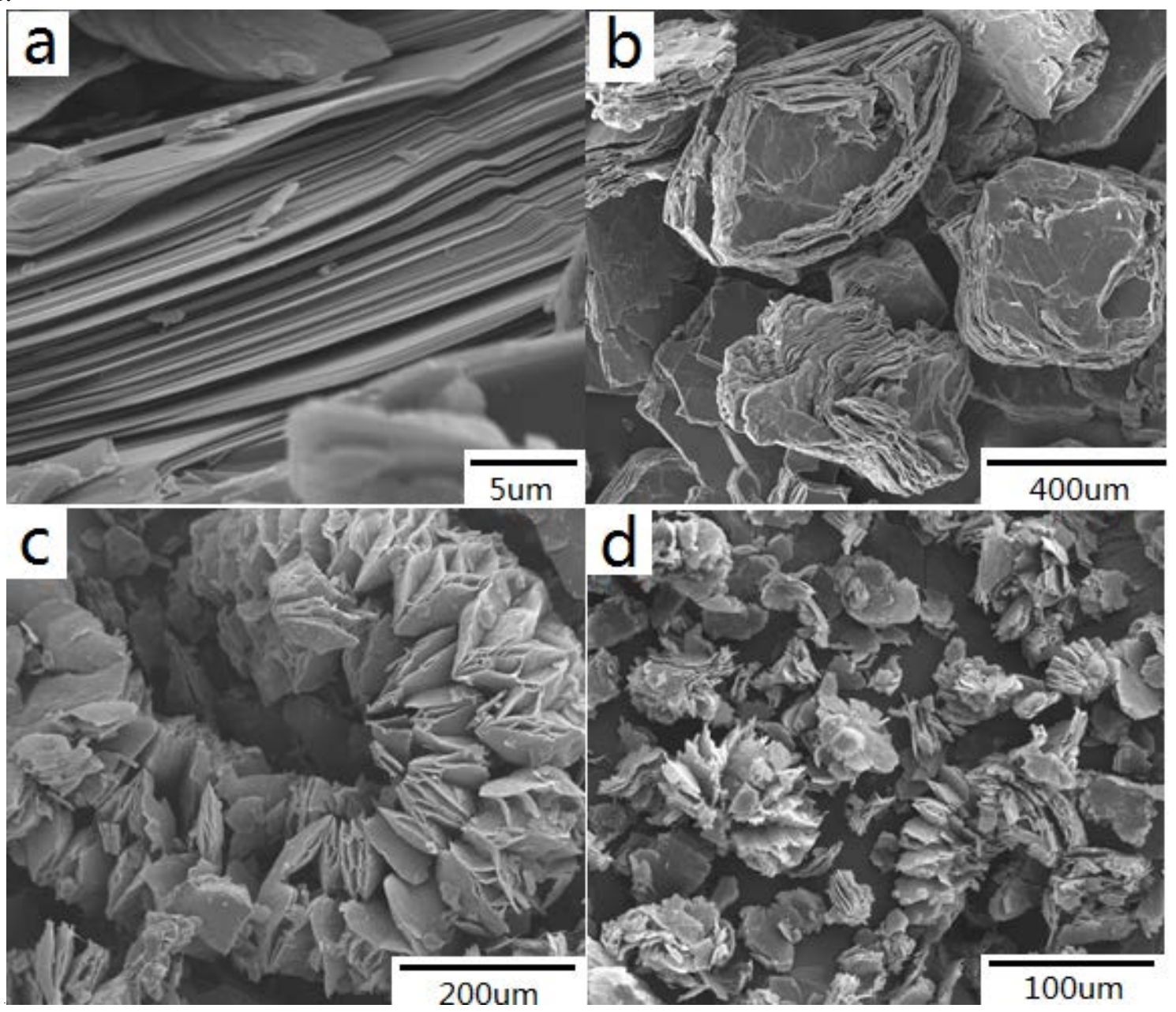

Fig.4 The morphology of nature graphite and expandable graphite. Nature graphite (a), "micro-expansion" expandable graphite (b), expansion graphite of hydrothermal method (c) and traditional chemical preparation method (d).

Fig.4(c) and (d) were expansion graphite of hydrothermal method and traditional chemical preparation method, respectively. It can be seen that the use of traditional oxidation method of expanded graphite, graphite crystal structure has been severely damaged, preparation of expanded graphite has lost its integrity, there is no 'worm' structure, but to make structural spread out. This kind of phenomenon is also related to the size of the flake graphite, this thesis used 100 mush small flake graphite as raw materials, due to the relatively small size of graphite and poor structural integrity, thus expansion volume is not high. And the structure of adopt hydrothermal preparation of expanded graphite was relatively complete, still can see the 'worm' structure, but expansion of expanded graphite were insufficient and flake graphite layer was not entirely, the layers of graphite was not completely "out" by high temperature steam completely "out", this showed that the hydrothermal method to continue to study.

\section{Conclusion}

The expandable graphite were prepared by hydrothermal, the optimum parameters were: $0.4 \mathrm{ml}$ of H2O2, $7 \mathrm{ml} \mathrm{H} 2 \mathrm{SO}$, the hydrothermal temperature is $100 \mathrm{oC}$ and hydrothermal time is $60 \mathrm{~min}$. The expansion volume achieve $160 \mathrm{ml} / \mathrm{g}$. Compared the traditional chemical method, the 
expandable graphite prepared by hydrothermal has reduced the dosage of $\mathrm{H} 2 \mathrm{O} 2$ and $\mathrm{H} 2 \mathrm{SO} 4$, decreased the peroxide of praphite, and obtain integrated 'worm' structure expansion graphite.

\section{Acknowledgement}

This research was financially supported by the National Science and Technology Support Program of China (No. 2013BAE04B02 and No. 2013BAE04B03).

\section{References}

[1] H. J. Duan, H. Q. Kang, W. Q. Zhang, X. Ji, Z. M. Li, J. H. Tang, Core-shell structure design of pulverized expandable graphite particles and their application in flame-retardant rigid polyurethane foams, Polym. Int. (2013) 232-246.

[2] G. Tang, R. Zhang, X. Wang, B. Wang, L. Song, Y. Hu, X. Gong, Enhancement of Flame Retardant Performance of Bio-Based Polylactic Acid Composites with the Incorporation of Aluminum Hypophosphite and Expanded Graphite, J. Macromole. Sci. Part A, 50 (2013) 255-269.

[3] L. Jihui, D. Huifang D, L. Qian, L. Shufen, Preparation of sulfur-free expanded graphite with $320 \mu \mathrm{m}$ mesh of flake graphite, Mater. Let. 60 (2006) 3927-3930.

[4] D. F. Liu, H. J. Lu, Z. D. Wu, J. Z. Liang, W. W. Tang, X. G. Chen, Ultrafine preparation process and influencing factors of expansible graphite flake graphite, Carb. technol. 02 (2013) 16-19.

[5] J. H. Li, L. L. Feng, Z. X. Jia, Preparation of expanded graphite with $160 \mu \mathrm{m}$ mesh of fine flake graphite, Materials Letters, 60 (2006) 746-749.

[6] Z. Ying, X. Lin, Y. Qi, J. Lou, Preparation and characterization of low-temperature expandable graphite, Mater. Res. Bull. 43 (2008) 2677-2686.

[7] C. F. Kuan,, K. C. Tsai, C. H. Chen, H. C. Kuan, T. Y. Liu, C. L. Chiang, Preparation of expandable graphite via H2O2-hydrothermal process and its effect on properties of high-density polyethylene composites, Polym. Comp. 33 (2012) 872-880.

[8] Y. L. Dong, G. J. Zhou, H. X. Ding, F. L. Yuan, The preparation technology and application of expanded graphite, J. Heilongjiang ShuiZhuan, 03 (2010) 59-63.

[9] Y. Zhu, X. X. Xia, Study of preparation and structure of expanded graphite, J. Shaanxi univers. sci. Technol. (Natural science edition), 01 (2011) 29-31. 